\title{
Future Oncology: a 10-year anniversary issue
}

\author{
"Although the past decade has provided many significant discoveries \\ across all specialties in oncology, the coming decade will again provide \\ many opportunities to advance our understanding and develop more \\ effective diagnostic methods and treatments."
}

\section{Nicholas J Ward ${ }^{*, 1} \&$ Gemma Westcott $^{1}$}

In the 10 years since our launch, the diverse field of oncology has changed dramatically, and Future Oncology has strived to present these developments from across the whole research spectrum. The past 10 years have seen remarkable developments in diagnostic and therapeutic approaches for treating cancer, and the rapid discovery and application of biomarkers and genomic medicine has facilitated personalized approaches that have revolutionized both patient's quality of life and life expectancy.

This special issue celebrates a decade since the launch of Future Oncology and is compiled with the help of members of the journal's editorial board and regular contributors. It aims to provide a broad representation of the progress made in particular areas of oncology, detailing the past, present and future possibilities in the diagnosis and treatment of several cancers. While we discuss only some of the articles contained in this issue in this Foreword, we encourage you to take a look at the remaining excellent content.

In a special report by Cédric Rébé and François Ghiringhelli (both INSERM UMR 866, France) [1], the authors explore the well-known fact that although chemotherapy kills cancerous cells, it can also impair the adaptive immune system's ability to combat the remaining cancerous cells. This article succinctly reviews the past and current situation of this area and proposes future strategies that are hoped will achieve the target of immunogenic cell death.

The article by Alba Brandes (IRCCS Institute of Neurological Sciences, Italy) and colleagues presents the authors perspective on the controversies surrounding the use of bevacizumab in the treatment of recurrent glioblastoma [2]. Recurrent glioblastoma is a challenging condition due to continuing poor prognosis and limited therapeutic options. Although approved by the US FDA, bevacizumab has not been approved by the EMA and, despite high response rates observed in clinical trials, there is ongoing debate regarding its impact on survival. The authors present evidence on several open issues. In regard to response evaluation, they identify that there is no recognized optimal criteria for response evaluation and subsequently argue that the relationship between response and survival is unclear. They go on to state that the optimal administration schedule for bevacizumab in glioblastoma is not known, along with the drug's efficacy as a third- or post-third-line therapy. With regards to patient selection, the authors show that more than $50 \%$ of patients do not respond to bevacizumab, and stipulate that urgent biomarker research is required to identify reliable biomarkers of response to bevacizumab.

The review 'Immunotherapy for NSCLC: the past 10 years' by Johan

'Future Medicine Ltd, Unitec House, 2 Albert Place,London, N3 1QB, UK

*Author for correspondence: Tel.: +44 (0) 208371 6090; n.ward@futuremedicine.com

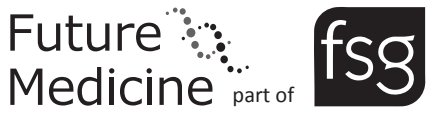

\section{KEYWORDS}

- biomarkers • diagnostics

- genomic medicine • oncology

- treatment

“The past 10 years have seen remarkable developments in diagnostic and therapeutic approaches for treating cancer, and the rapid discovery and application of biomarkers and genomic medicine has facilitated personalized approaches that have revolutionized both patient's quality of life and life expectancy." 
Vansteenkiste (University Hospitals KU Leuven, Belgium) and colleagues summarizes the progress made in the past decade and highlights important clinical trials and potential future developments [3]. The authors remind us that lung cancer remains the leading cause of cancerrelated deaths, with NSCLC accounting for 85\% of these. Recent developments in the understanding of the immune system have facilitated the increase of clinical trials seen in this field. The authors summarize the two main approaches in NSCLC; antigen-specific therapeutic cancer vaccination and nonantigen-specific immunotherapy, which includes immune checkpoint inhibitors. Evidence is presented from the MAGRIT trial [4], demonstrating that vaccination in NSCLC, at the present time, does not work. Data regarding the efficacy of immune checkpoint inhibitors are presented, showing the great potential of this treatment modality. In fact, there are currently several Phase III trials focused on checkpoint inhibitors as first-line treatments for advanced NSCLC.

An interesting article presented by Michaël Grynberg (Hôpital Jean Verdier, France) and colleagues focuses on fertility preservation in cancer patients, detailing the past, present and future possibilities [5]. The authors cover fertility preservation in both sexes in extensive detail. Fertility preservation should be addressed in patients of all ages, especially where current first-line treatments such as chemotherapy, radiotherapy and surgery are likely to alter an individual's natural fertility. Detail on the importance of oncofertility counseling as part of the management of younger patients and the need for increased oncologist knowledge in this area are key points of this article.

Another review that is featured in this issue focuses on muscle-wasting that occurs in cancerrelated cachexia syndrome [6]. Contributed by Giovanni Mantovani (University of Cagliari, Italy) and colleagues, the article presents an overview of the pathophysiology of this condition, examining the mechanisms involved in its development and progression. The authors explain that there are no available, efficacious treatments for cancer cachexia-related muscle wasting and state that treatments targeting upor downstream pathways in skeletal muscle are essential. Potential pharmacological treatments for the future are the focus of the latter paragraphs of the article. Nonsteroidal anti-inflammatory drugs are presented as possible future treatment modalities; however, the evidence at this stage indicates that although improving several other symptoms, these drugs have limited effects on muscle mass. Anticytokine drugs are proposed as one of the most promising modalities in etiopathogenetic therapy of muscle wasting in cancer cachexia. The authors conclude that multimodal approaches can be effective for general cancer cachexia management, whereas pharmacological agents are best suited for the muscle wasting aspect of this condition.

Giuseppe Aprile (University and General Hospital Udine, Italy) and colleagues contribute an editorial documenting the development and future possibilities in the treatment of advanced colorectal cancer [7]. The authors present the situation prior to 2010, with great advances in overall survival due to the introduction of novel cytotoxic agents and combination therapies. They go on to present the achievements of the past 5 years and the innovations driving the progress in the field, such as the increased understanding of the molecular basis of the disease. The authors state that future progress depends on developing the understanding of tumor biology and identifying mechanisms of treatment resistance. The role of immunotherapy in the treatment of colorectal cancer is also proposed as a key area for future investigation.

The Editorial article provided by Ismail Jatoi (University of Texas Health Science Center, TX, USA), 'Breast cancer screening: past, present and future', presents the counterintuitive argument that improvements in breast cancer therapy and screening technology are actually likely to be reducing the overall benefit of mammography screening [8]. In regard to future approaches, Jatoi suggests consideration of 'less might be better', in terms of using less sensitive screening methods to prevent overdiagnosis. The introduction of screening clinical breast examination as an alternative to mammography is also suggested and evidence of comparable results in clinical trials is presented.

Other excellent contributions to the issue include the latest developments in cancer endocrinology [9], a commentary on rare ocular tumors [10], a look at gastric cancer over the last two decades [11], an editorial documenting the increasing role of chemotherapy in prostate cancer [12], an overview of image-guided brachytherapy in cervical cancer [13] and reviews documenting the evolving landscape in precision medicine for colorectal cancer [14] and global developments in stereotactic body radiotherapy [15]. 
Although the past decade has provided many significant discoveries across all specialties in oncology, the coming decade will again provide many opportunities to advance our understanding and develop more effective diagnostic methods and treatments. The commissioning editors would like to thank all contributors over the past 10 years for making Future Oncology a success, and we look forward with excitement to the next decade.

\section{Financial \& competing interests disclosure}

The authors are employees of Future Science Group. The authors have no other relevant affiliations or financial involvement with any organization or entity with a financial interest in or financial conflict with the subject matter or materials discussed in the manuscript apart from those disclosed.

No writing assistance was utilized in the production of this manuscript.

\section{References}

1 Rébé C, Ghiringhelli F. Cytotoxic effects of chemotherapy on cancer and immune cells: how can it be modulated to generate novel therapeutic strategies? Future Oncol. 11(19), 2645-2654 (2015).

2 Franceschi E, Bartolotti M, Brandes A. Bevacizumab in recurrent glioblastoma: open issues. Future Oncol. 11(19), 2655-2665 (2015).

3 Vansteenkiste J, Craps J, De Brucker N, Wauters I. Immunotherapy for non-small-cell lung cancer: the past 10 years. Future Oncol. 11(19), 2681-2695 (2015).

4 Vansteenkiste JF, Cho BC, Vanakesa T et al. MAGRIT, a double-blind, randomized, placebo-controlled Phase III study to assess the efficacy of the recMAGE-A3 + AS15 cancer immunotherapeutic as adjuvant therapy in patients with resected MAGE-A3-positive non-small cell lung cancer (NSCLC). Ann. Oncol. 25(Suppl. 4), Abstract 1173 O (2014).
5 Sonigo C, Sermondade N, Benard J et al. The past, present and future of fertility preservation in cancer patients. Future Oncol. 11(19), 2667-2680 (2015).

6 Madeddu C, Mantovani G, Gramignano G, Astara G, Macciò A. Muscle wasting as main evidence of energy impairment in cancer cachexia: future therapeutic approaches. Future Oncol. 11(19), 2697-2710 (2015).

7 Aprile G, Rihawi K, Scartozzi M, Bordonaro R. Steps ahead in the treatment of advanced colorectal cancer: past, current, and possible future scenarios. Future Oncol. 11(19), 2625-2628 (2015).

8 Jatoi I. Breast cancer screening: past, present and future. Future Oncol. 11(19), 2621-2624 (2015).

9 Berstein LM. Cancer endocrinology: noticeable developments in the last decade. Future Oncol. 11(19), 2617-2620 (2015).

10 Sagoo MS, Filipovic A, Al Harby L, Stebbing J. Rare tumours: an eye on the future. Future Oncol. 11(19), 2641-2644 (2015).
11 Aprile G, Silvestris N, Antonuzzo L, Scartozzi M. Metastatic gastric cancer in the last two decades: goals achieved and future promises. Future Oncol. 11(19), 2633-2636 (2015).

12 Di Lorenzo G, Sonpavde G. The expanding role of chemotherapy in prostate cancer. Future Oncol. 11(19), 2637-2640 (2015).

13 Trifiletti DM, Showalter TN. Image-guided brachytherapy in cervical cancer: past, present and future. Future Oncol. 11(19), 2629-2632 (2015).

14 Fisher KW, Lopez-Beltran A, Montironi R, Cheng L. Precision medicine in colorectal cancer: evolving genomic landscape and emerging consensus. Future Oncol. 11(19), 2711-2719 (2015).

15 Lo SS, Slotman BJ, Lock M et al. The development of stereotactic body radiotherapy in the past decade: a global perspective. Future Oncol. 11(19), 2721-2733 (2015). 